\title{
2019 Lasker Basic Research Award celebrates immunologists Jacques Miller and Max Cooper
}

\begin{abstract}
The Lasker Foundation recognizes Jacques Miller (The Walter and Eliza Hall Institute of Medical Research) and Max D. Cooper (Emory University School of Medicine) with 2019's Albert Lasker Basic Medical Research Award for demonstrating that B and $\mathrm{T}$ cells represent distinct compartments of the adaptive immune system (Figure 1). Their discoveries laid the foundations for modern immunology and groundbreaking medical advances, including monoclonal antibody therapeutics and immunotherapy.

In 1960, much of immunology was embroiled in controversy, but researchers of adaptive immunity united over some key principles: Plasma cells were the source of antibodies, proteins with important roles in controlling infection. Lymphocytes could be identified in lymphoid tissues such as the spleen, lymph nodes, and the thymus. However, though immunologists had clearly demonstrated the spleen's and lymph nodes' contributions to immune function, the thymus was considered a vestigial lymphatic organ: thymectomy, at least in adult animals, had no discernible effect on immune processes.
\end{abstract}

\section{Vestigial no more}

As a $\mathrm{PhD}$ student at the University of London, Jacques Miller became intrigued by a form of virus-induced murine lymphocytic leukemia originating in the thymus. New reports had revealed that the disease could be transferred to a leukemia-resistant strain of mice by injecting healthy animals with extracts from a sick animal's leukemic tissues. But there was a twist, as Miller explains: "The filtrate had to be injected into newborn mice. If it was injected into adult mice, they would not get leukemia. That interested me."

Miller hypothesized that the leukemia-causing virus could only multiply in the newborn thymus, which undergoes involution with age, decreasing in size and cell number. He tested this hypothesis by removing the thymus from new-
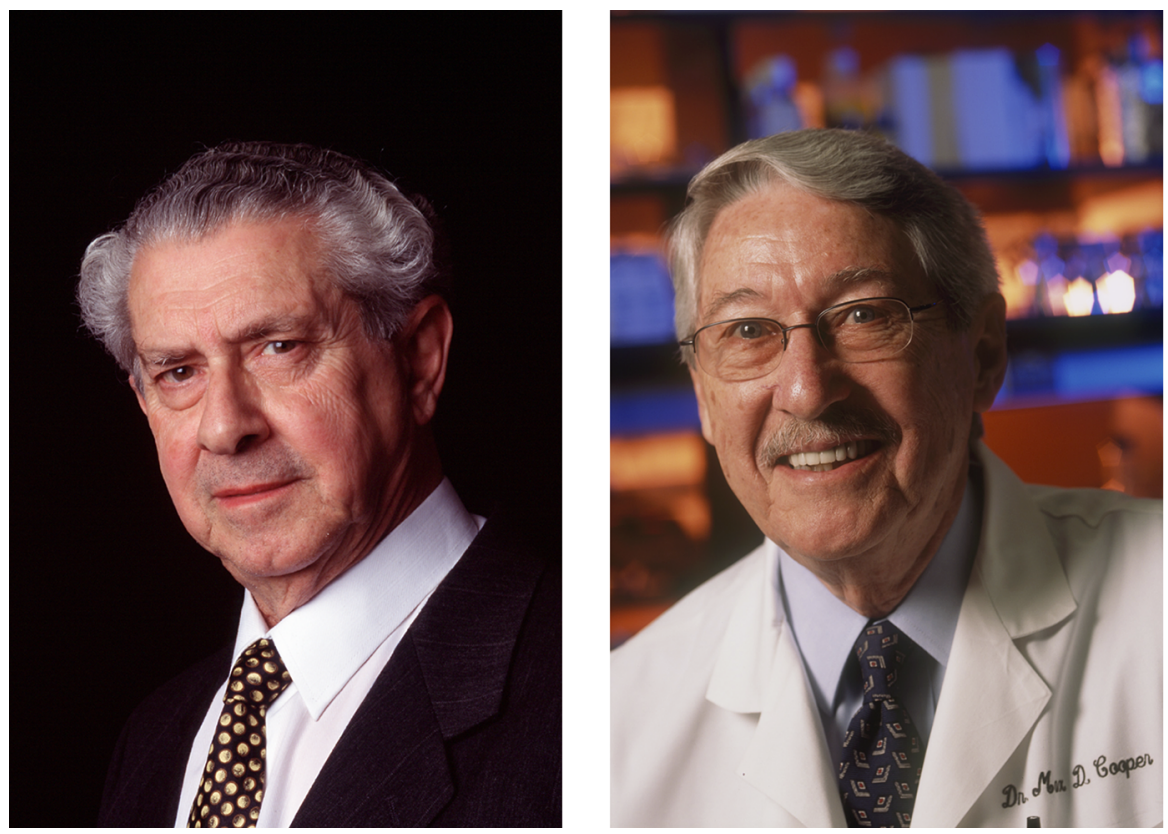

Figure 1. 2019 Lasker Basic Medical Research Award recipients Jacques Miller (left) and Max D. Cooper (right). The pair have been recognized for their discovery that T and B lymphocytes represent distinct compartments of adaptive immunity. Dr. Miller's photo appears courtesy of The Walter and Eliza Hall Institute of Medical Research. Dr. Cooper's photo appears courtesy of Georgia Research Alliance/Billy Howard.

born mice, injecting virus, and regrafting the thymus later in life. Unexpectedly, however, he discovered that thymectomy in the neonatal period resulted in the adult mice having extremely poor health, even if not previously injected with the leukemic virus.

"I did a postmortem and saw lesions in the liver, which suggested that the mice had been infected by hepatitis virus," Miller recalls. "I saw that the lymphoid system was atrophic. There were not many lymphocytes in the circulation. There were not many lymphocytes in the lymphoid tissues. That made me wonder, 'What's happening?"

Miller tested immune responses in the neonatally thymectomized mice and found that beyond being infection prone, these mice could not reject skin grafts from other mouse strains or even rats. They also displayed impaired antibody responses to some injected antigens. These findings indicated that the thymus plays a key role in early immune development that affects lifelong immunity $(1,2)$.

Miller's conclusions challenged the era's dogma that the thymus had no apparent function. They were not immediately accepted by the scientific community. Although the outcomes of neonatal thymectomy were clear, critics argued that contamination of the University of London's animal facilities, which were located in a converted horse stable, produced lifelong immune impairment in the mice. On an Eleanor Roosevelt fellowship, Miller traveled to the US and repeated the experiments using the NIH's germ-free mice. In sterile conditions, the thymectomized mice were spared the infectious consequences of immunodeficiency but remained unable to reject skin grafts from rats, confirming Miller's interpretation that the thymus has a critical role in immune function. 


\section{Clinical conundrums and clarity}

Miller's findings were well received in Robert Good's lab at the University of Minnesota, where researchers had observed similar phenomena. Soon after Miller's initial reports on thymic function, Max Cooper joined Good's team with the goal of teasing apart the biology underlying congenital immune deficiency diseases.

Cooper began his career as a pediatrician trained in clinical allergy and immunology, but was drawn to immunology research in response to the challenging clinical conundrums posed by immunodeficient patients: "I quickly realized that if I was going to contribute anything original to their care, I would have to start all over again." Cooper decided to pursue basic immunology research under the guidance of Dr. Good, an esteemed immunologist and clinician who would become most famous for performing the first non-twin bone marrow transplant. "Good's environment was a good fit for me because he was interested in immune system development, immunodeficiency diseases, evolution, transplantation, the complement system, lymphoproliferative diseases, and autoimmunity."

"The prevailing viewpoint then was that circulating lymphocytes derived from the thymus were converted into plasma cells following antigen stimulation" Cooper explains. However, discordant clinical characteristics of boys with X-linked agammaglobulinemia and Wiskott-Aldrich syndrome suggested that lymphopenia and immunodeficiency could be uncoupled from defective antibody production. A block in the lymphocyte to plasma cell conversion could explain X-linked agammaglobulinemia, but the profound lymphocyte deficiency and thymic atrophy in contrast with plasma cell and immunoglobulin abundance he observed in WiskottAldrich patients argued against the single lymphocyte lineage hypothesis.

Cooper focused on an avian model to examine this paradox. In addition to the thymus, birds have the bursa of Fabricius, a hindgut lymphoid organ that had been shown to contribute to antibody production (3). Subsequent studies suggested that the thymus and bursa had different roles in the development of cellular and humoral immunity, but the effects of early thymectomy versus bursectomy differed across laboratories and chicken strains.
Hoping to clarify the roles of the thymus and bursa, Cooper performed combinations of bursectomy and thymectomy in newly hatched chicks and then exposed them to near-lethal levels of whole-body irradiation to eliminate any lymphocytes generated before hatching. Evaluation of immunological integrity weeks after recovery from irradiation yielded unambiguous results: bursectomized and heavily irradiated chickens had normal thymus development and intact cellular immunity, but were completely devoid of germinal centers, plasma cells, and immunoglobulins. In contrast, thymectomized and irradiated chickens were primarily deficient in small lymphocytes and cellular immune functions; they made germinal centers, plasma cells, and immunoglobulins, with moderately impaired specific antibody responses $(4,5)$. The results clearly supported a dual lineage model of lymphocyte differentiation that elucidated the pathogenesis of immunodeficiency diseases, including agammaglobulinemia and Wiskott-Aldrich syndrome, and aligned with results Miller and others had observed in mammals.

\section{Illuminating distinct lymphocyte origins}

Distinguishing the functions of the thymus and bursa kicked immunologists into high gear. Cooper recalls, "We learned from the Till and McCulloch group that stem cells gave rise to all the different blood cell types. Others showed that hematopoietic stem cells gave rise to cells populating the thymus, and then Moore and Owen showed in birds that lymphoid cells in the thymus and bursa came from circulation. That information allowed us to construct a working model of how hematopoietic stem cells give rise to the thymus-derived and bursa-derived lymphocyte lineages."

Miller and his first PhD student, Graham Mitchell, swiftly revealed that thymus-derived cells had a distinct function: "At that time, we thought that thymus-derived cells, or $\mathrm{T}$ cells, were the cells producing the most antibodies and mediating foreign graft rejection," he says. "We used an experimental system where we injected bone marrow as well as thymus-derived lymphocytes into an irradiated mouse, and we showed that antibody-forming cell precursors came from the bone marrow, whereas cells responsible for rejection of skin grafts or virus-infected cells came from the thymus."

The findings, published in 1968 (6, 7 ), elucidated the emerging model of thymus- and bursa-specific functions, delineating two distinct populations of thymus-derived and bursa- or bone marrow-derived cells, which we know today as $\mathrm{T}$ and B cells. Following Miller and Mitchell's reports, an explosion of immunological discovery further clarified functions and interactions of $\mathrm{T}$ and B lymphocytes. In 1974, John Owen, Martin Raff, and Cooper, working at University College London, demonstrated that $B$ cells could be generated in cultures of fetal liver and in adult bone marrow (8).

\section{From foundations to breakthroughs}

Following these seminal discoveries, Miller and Cooper pursued lines of investigation that established additional groundwork for clinically significant advances in immunology. For recent generations of scientists, it's likely difficult to envision studying immunology before this modern understanding of the adaptive immune system. Cooper agrees: "Once you know something it seems as if you always knew it."

Though officially retired, Jacques Miller's tenacious interest in new areas of immunology keeps him involved at the Walter and Eliza Hall Institute. The microbiome and advances in immunotherapy, particularly those involving Tregs, hold his interest as particularly exciting areas of progress. Of being recognized with the Lasker Award, he says: "I am very, very honored and I'm humbled to get it. And, of course, I'm delighted."

Max Cooper's work continues at Emory University. His exploration of adaptive immunity's evolutionary origins revealed that the basic genetic program for prototypic $\mathrm{T}$ and $\mathrm{B}$ cell lineages was already present in a common vertebrate ancestor approximately 500 million years ago; however, antibodies made by jawless vertebrates have unique structural properties and specificities that could be leveraged for biomedical purposes (9). But he says that recognizing the adaptive immune system's compartmentalization remains one of the most exciting times of his entire life. 
When he learned that he and Miller would receive the Lasker award, "I was really surprised and utterly delighted, of course. It's especially meaningful to me in that it recognizes our work and all the people that worked together."

\section{Elyse Dankoski}

1. Miller JF. Immunological function of the thymus. Lancet. 1961;2(7205):748-749.

2. Miller JF. Role of the thymus in immunity.
Br Med J. 1963;2(5355):459-464.

3. Glick B, Chang TS, Jaap RG. The bursa of Fabricius and antibody production. Poultry Science. 1956;35(1):224-225.

4. Cooper MD, Peterson RD, Good RA. Delineation of the thymic and bursal lymphoid systems in the chicken. Nature. 1965;205:143-146.

5. Cooper MD, Raymond DA, Peterson RD, South MA, Good RA. The functions of the thymus system and the bursa system in the chicken. J Exp Med.1966;123(1):75-102.

6. Miller JF, Mitchell GF. Cell to cell interaction in the immune response. I. Hemolysin-forming cells in neonatally thymectomized mice recon- stituted with thymus or thoracic duct lymphocytes. J Exp Med. 1968;128(4):801-820.

7. Mitchell GF, Miller JF. Cell to cell interaction in the immune response. II. The source of hemolysin-forming cells in irradiated mice given bone marrow and thymus or thoracic duct lymphocytes. JExp Med.1968;128(4):821-837.

8. Owen JJ, Cooper MD, Raff MC. In vitro generation of B lymphocytes in mouse foetal liver, a mammalian 'bursa equivalent'. Nature. 1974;249(455):361-363.

9. Hirano M, et al. Evolutionary implications of a third lymphocyte lineage in lampreys. Nature. 2013;501(7467):435-438 